\title{
Convection and observable properties of late-type giants
}

\author{
A. Kučinskas ${ }^{1,2}$, H.-G. Ludwig ${ }^{3}$ and P.H. Hauschildt ${ }^{4}$ \\ ${ }^{1}$ National Astronomical Observatory of Japan, Mitaka, Tokyo 181-8588, Japan \\ email: arunas.kucinskas@nao.ac.jp \\ ${ }^{2}$ Institute of Theoretical Physics and Astronomy, Goštauto 12, Vilnius 01108, Lithuania \\ email: ak@itpa.lt \\ ${ }^{3}$ GEPI - CIFIST, Observatoire de Paris-Meudon, 5 place Jules Janssen, \\ 92195 Meudon Cedex, France \\ ${ }^{4}$ Hamburger Sternwarte, Gojenbergsweg 112, 21029 Hamburg, Germany
}

\begin{abstract}
We show that contrary to what is expected from 1D stationary model atmospheres, 3D hydrodynamical modeling predicts a considerable influence of convection on the spectral properties of late-type giants. This is due to the fact that convection overshoots into the formally stable outer atmospheric layers producing a notable granulation pattern in the 3D hydrodynamical models, which has a direct influence on the observable spectra and colors. Within the framework of standard 1D model atmospheres the average thermal stratification of the 3D hydro model can not be reproduced with any reasonable choice of the mixing length parameter and formulation of the turbulent pressure. The differences in individual photometric colors - in terms of $3 \mathrm{D}$ versus $1 \mathrm{D}$ - reach up to $\sim 0.2 \mathrm{mag}$, or $\Delta T_{\text {eff }} \sim 70 \mathrm{~K}$. We discuss the impact of full 3D hydrodynamical models on the interpretation of observable properties of late-type giants, briefly mentioning problems and challenges which need to be solved for bringing these models to a routine use within the astronomical community in 5-10 years from now.
\end{abstract}

Keywords. Convection; Hydrodynmaics; Stars: late-type, atmospheres, fundamental parameters, evolution

\section{Introduction}

Convection plays an important role in governing the interior structure and evolution of late-type giants (i.e., stars on the red and asymptotic giant branches, RGB/AGB). Besides of aiding the energy transport from stellar interiors to the outer layers, convection is important in delivering heavy elements from the nuclear burning layers to the outer atmosphere. Since convective mixing changes the chemical composition both in the outer atmosphere and stellar interiors, it eventually alters also the stellar structure because of changes in atomic and molecular opacities. This affects observable properties of a star, lifetimes in different evolutionary stages, and so forth. Obviously, proper understanding of convection is of fundamental importance for building realistic evolutionary models, which form the basis of our understanding of individual stars and stellar populations.

Convection in current theoretical models is treated in a rather simplistic way, typically within a framework of mixing length theory (MLT). Inevitably, this has a number of drawbacks. For instance, the efficiency of convection within the framework of MLT is scaled by a-priori unknown mixing-length parameter, $\alpha_{\mathrm{MLT}}$ (defined as the ratio of the mixing length to the pressure scale height), usually calibrated with Solar models. Almost certainly, $\alpha_{\mathrm{MLT}}$ needs not to be the same in main-sequence stars, subgiants, giants and supergiants, as is assumed in current evolutionary models (see, e.g., Freytag \& Salaris 1999 for more details on this issue). Not surprisingly, there have been many attempts 


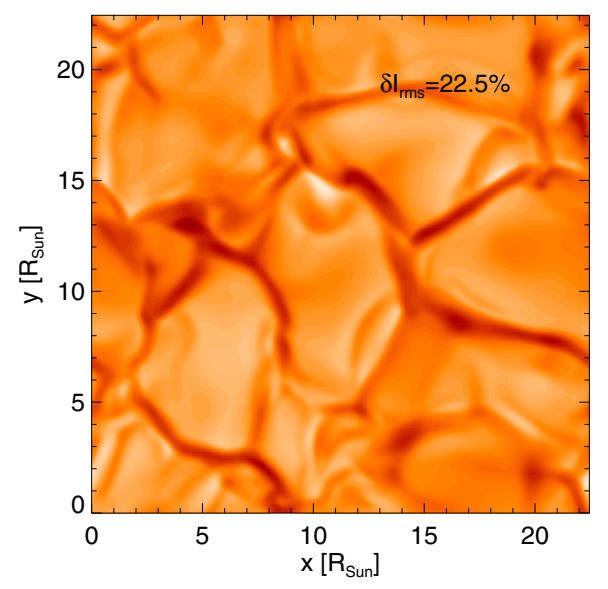

Figure 1. Snapshot of the emergent white light intensity during the temporal evolution of a hydrodynamical red giant model. Note the spatial scales of the granulation pattern. The relative rms intensity contrast of the granulation pattern is $22.5 \%$ at this particular instant in time.

during the last few decades to improve the treatment of convection in stellar models (for example, implementing the concept of convective overshooting, which allows for convection to penetrate beyond the classical boundaries of a convective layer). While such efforts are incremental steps towards a more realistic modeling of convection in stellar interiors, a fundamental breakthrough in this area is likely to go beyond the classical stationary 1D modeling. Obviously, this may be possible with full 3D hydrodynamical models, as they account for time-dependent and three-dimensional character of convection from first principles, providing a degree of realism in the treatment of non-stationary phenomena (and convection in particular) that is beyond reach with classical approach.

\section{Convection in late-type giants with $3 \mathrm{D}$ hydrodynamical models}

To investigate the influence of convection on the observable properties of late-type giants we employed a radiation-hydrodynamics code named $\mathrm{CO}{ }^{5} \mathrm{BOLD}$ (mainly developed by B. Freytag and M. Steffen; for a description of the code see Wedemeyer et al. 2004) to construct a fully time-dependent 3D hydrodynamical model of a prototypical latetype giant $\left(T_{\text {eff }} \simeq 3700 \mathrm{~K}, \log g=1.0,[\mathrm{M} / \mathrm{H}]=0.0 ;\right.$ Fig. 1$)$. The final goal of this work was to make a comparison of the thermal structures and spectral properties of the $3 \mathrm{D}$ model with those predicted by classical 1D model atmospheres. Note that 1D atmosphere model used in this comparison was calculated using the same atmospheric parameters and employed the same physical input data (opacities, equation of state, description of radiative transfer) as the hydrodynamical model.

Indeed, the thermal structure of the 3D model is quite different from that obtained with the classical 1D approach (Fig. 2). Three 1D models shown in Fig. 2 were calculated for different mixing length parameters $\left(\alpha_{\mathrm{MLT}}=1.0,1.5,2.0\right)$ assuming a vanishing turbulent pressure, i.e. $P_{\text {turb }}=0$. Two further $1 \mathrm{D}$ models were constructed including a non-zero turbulent pressure, calculated as $P_{\text {turb }}=f \rho v^{2}$, where $\rho$ and $v$ are gas density and velocity respectively, and $f$ is a dimensionless factor (usually $f<1$ ). Interestingly, no reasonable combination of $\alpha_{\mathrm{MLT}}$ and $f$ allows to construct a classical $1 \mathrm{D}$ model which reproduces the structure of 3D hydrodynamical model (Fig. 2). In the framework of MLT convection is confined to optically thick regions $(\log P>3.5)$, and consequently the optically thin 


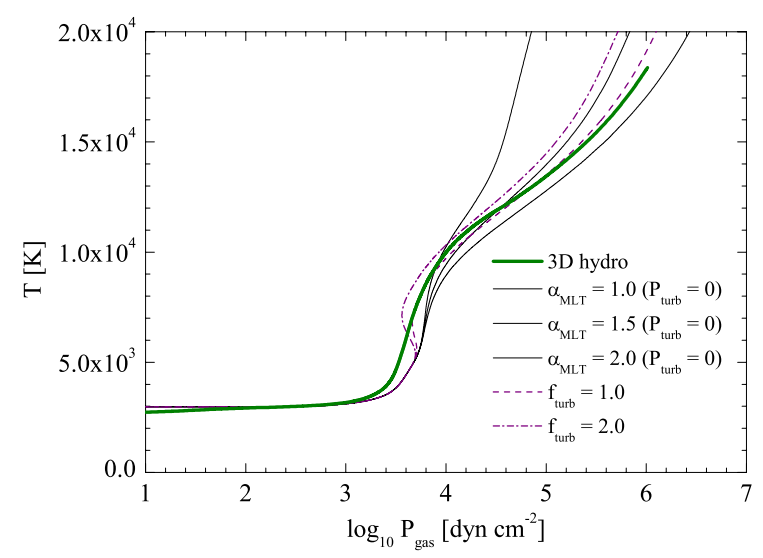

Figure 2. Temperature stratification in the 3D hydrodynamical model of a late-type giant, as a function of gas pressure, $P_{\text {gas }}$. Thick solid line is a 3D hydrodynamical model (averaged on surfaces of equal geometrical depth), thin lines are 1D plane-parallel models calculated using different mixing-length parameter $\left(\alpha_{\mathrm{MLT}}=1.0,1.5,2.0\right.$, from left to right; in all cases $\left.P_{\text {turb }}=0\right)$. Two 1D models with non-zero turbulent pressure are given by dashed $\left(\alpha_{\mathrm{MLT}}=2.0\right.$ and $\left.f=1.0\right)$ and dashed-dotted $\left(\alpha_{\mathrm{MLT}}=2.0\right.$ and $\left.f=2.0\right)$ lines.

regions are hardly sensitive to convection. However, the geometric distance between the upper boundary of the convective region and optical depth unity is not large, and in the 3D hydrodynamical model convection overshoots into the optically thin layer. This produces a prominent granulation pattern on the stellar surface (cf. Fig. 1) which has a direct influence on the emerging spectrum, and thus - the photometric colors.

The differences between colors predicted with 3D and 1D models are significant, e.g., $\Delta(V-K) \sim 0.2$, or $\Delta T_{\text {eff }} \sim 70 \mathrm{~K}$ (Fig. 3; see Kučinskas et al. 2005 for details on the calculation of spectra and colors with the $3 \mathrm{D}$ model). Note that this difference is comparable to a typical error margin in the individual $T_{\text {eff }}$-color relations (Kučinskas et al. 2006) which are used to transform theoretical isochrones to color-magnitude planes.

\section{Conclusions}

Clearly, our 3D model of a prototypical late-type giant predicts considerably different thermal structures from those inferred with the classical 1D model atmospheres. Spectral properties of the $3 \mathrm{D}$ model are rather sensitive to convection too, which results in significant differences between photometric colors calculated with the 3D and 1D model atmospheres. All this should be properly taken into account both with evolutionary models and model atmospheres of late-type giants. At the same time, there is a number of issues that will have to be tackled to improve the 3D models, just to name a few:

- Implement a possibility of direct spectral synthesis with 3D stellar atmosphere models, preferably in non-LTE;

- Improve spectral line databases, atomic and (especially!) molecular opacities;

- Investigate the properties of stars in the regions of HR diagram that were poorly covered with 3D models up to now, at different metallicities (especially RGB/AGB stars).

While 3D hydrodynamical models are still computationally expensive today, grids of synthetic spectra calculated in 3D approach may be available in 5-10 years from now (see Ludwig \& Kučinskas 2005 for a discussion). No doubt, this will open new possibilities for improving models of stellar evolution (especially those on RGB/AGB), and will alow to study a variety of new phenomena that are beyond reach with classical 1D models. 


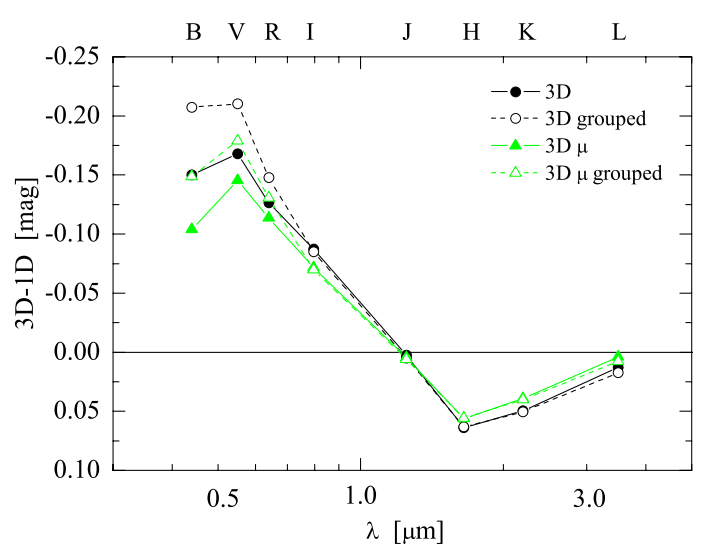

Figure 3. Influence of surface granulation on the broad-band photometric colors of red giant, as reflected by magnitude differences in various band-passes (indicated on the top of the panel) between the predictions of 3D hydrodynamical and classical 1D model atmospheres. The different lines depict various approximations employed in the treatment of the 3D radiative transfer (see Kučinskas et al. 2005 for details).

\section{References}

Freytag, B. \& Salaris, M. 1999, ApJ 513, L49

Kučinskas, A., Hauschildt, P.H., Ludwig, H.-G. et al. 2005, A\&A 442, 281

Kučinskas, A., Hauschildt, P.H., Ludwig, H.-G. et al. 2006, this volume

Ludwig, H.-G. \& Kučinskas, A. 2005, in: F. Favata (ed.), Cool Stars, Stellar Systems and the Sun 13, ESA SP-560, p. 319

Wedemeyer, S., Freytag, B., Steffen, M. et al. 2004, A\&A 414, 1121

\section{Discussion}

FEAST: At least in case of large amplitude variables one can circumvent the models by empirical calibration of luminosity, age and metallicity in our Galaxy and the Local Group.

KuČInsKas: Calibration of selected stellar properties is indeed possible with observations. However, all three quantities you mention are derived employing certain theoretical assumptions, thus they are not entirely model-independent.

FEAST: What is it in your models which determines the size of the convective cells?

KUČINSKAS: To first approximation the size of the convective cells is set by (five to ten times) the pressure scale height at the stellar surface. In earlier spectral types with more intense radiation fields the cell size is somewhat biased towards larger values. Sphericity related effects in late-type supergiants are not fully clarified yet. However, there exist indications of a bias again towards larger cells.

ARDEBERG: With the new synthetic spectra and colors available, should we foresee any problems related to our current models, simulations and conclusions?

KUČINSKAS: I would expect that predictions of the current theoretical models will not change drastically. There is no doubt, however, that certain details of the global picture will be different. How much - this needs to be clarified with further modeling. 